hence the line $y=0, x+z=0$ is a torsal generator and $x+z=0$ is the torsal plane. The simple conic passes through the point of intersection of the three double conics and touches the generator $x=0, y=0$.

If $f+g=0$ the conic will be factorable. The rectilinear directrix is

$$
x+z=0, \quad h z-f w=0 .
$$

If the simple conic be required to intersect $c_{3}^{\prime}$ in a second point, the former must break up.

When the scroll belongs to a linear complex, the complex must be special. A quintic scroll which has three double conics which touch the same line at the same point, does not exist.

Every quintic scroll having three double conics contains either a simple conic or a simple directrix. If in the former case the planes of all four conics pass through the same point, then the plane of the simple conic contains three generators passing through that point.

CorNell UNIVERsity,

October 10, 1902.

\title{
SURFACES REFERRED TO THEIR LINES OF LENGTH ZERO.
}

BY DR. L. P. EISENHART.

(Read before the American Mathematical Society, October 25, 1902.)

Consider a surface $S$ referred to a general system of parametric lines, $u=$ const., $v=$ const. Denote by $x, y, z$, the cartesian coördinates of a point on $S$ and $X, Y, Z$, the direction cosines of the normal to the surface. As usual, we put

(1) $E=\sum\left(\frac{\partial x}{\partial u}\right)^{2}, \quad F=\sum \frac{\partial x}{\partial u} \frac{\partial x}{\partial v}, \quad G=\sum\left(\frac{\partial x}{\partial v}\right)^{2}$,

and

(2) $D=\sum X \frac{\partial^{2} x}{\partial u^{2}}, \quad D^{\prime}=\sum X \frac{\partial^{2} x}{\partial u \partial v}, \quad D^{\prime \prime}=\sum X \frac{\partial^{2} x}{\partial v^{2}}$ 
When the parameter curves are the lines of length zero,

$$
E=G=0,
$$

and the Codazzi equations * reduce to the simple forms

$$
\frac{\partial D}{\partial v}-D^{\prime} \frac{\partial}{\partial u} \log \frac{D^{\prime}}{F}=0,
$$

$$
\frac{\partial D^{\prime \prime}}{\partial u}-D^{\prime} \frac{\partial}{\partial v} \log \frac{D^{\prime}}{F}=0 .
$$

The expression for the mean curvature $\nmid$ becomes

$$
\frac{1}{2}\left(\frac{1}{\rho_{1}}+\frac{1}{\rho_{2}}\right)=-\frac{D^{\prime}}{F} \text {. }
$$

From (3) it follows that when $S$ is a surface of constant mean curvature, $D$ is a function of $u$ alone and $D^{\prime \prime}$ of $v$ alone. Conversely, when this condition is satisfied, either $D^{\prime}=0$ or $D^{\prime} / F$ is constant. In either case the mean curvature is constant; in the former, $S$ is a minimal surface. Hence

Theorem I. Surfaces of constant mean curvature are characterized by the property that, when the parametric lines are of length zero, $D$ is a function of $u$ alone and $D^{\prime \prime}$ of $v$ alone.

When any surface is referred to its lines of length zero, the directions of the lines of curvature are given by the equation $\ddagger$

$$
D d u^{2}-D^{\prime \prime} d v^{2}=0 \text {. }
$$

In consequence of the above results we have

THEOREM II. The lines of curvature on a surface of constant mean curvature are given by quadratures when the surface is referred to its lines of curvature.

By Bonnet's theorem $\S$ we know that there is associated with every surface of constant mean curvature, different from zero, a parallel surface of constant total curvature and conversely. Moreover, it can be seen from the general relations || which

* Bianchi, Lezioni, p. 91.

† Ibid., p. 104.

+ Ibid., p. 99.

\& Darboux, Leçons, vol. 3., p. 375.

\| Knoblauch, Einleitung in die allgemeine Theorie der krummen Flächen, p. 234-6. 
obtain between the fundamental coefficients of a surface and its parallel that lines of length zero and lines of curvature upon a surface of constant mean curvature correspond to asymptotic lines and lines of curvature respectively upon the parallel surface of constant total curvature. Hence

THEOREM III. The lines of curvature on a surface of constant total curvature are given by quadratures, when the surface is referred to its asymptotic lines.

Theorem II is not restricted to surfaces of constant mean curvature, but, as we shall see, is true of all isothermic surfaces. Let $S$ be an isothermic surface; then its linear element can be brought to the form

$$
d s^{2}=\lambda\left(d u_{1}^{2}+d v_{1}^{2}\right)
$$

where $\lambda$ is a function of $u_{1}$ and $v_{1}$, and the curves $u_{1}=$ const., $v_{1}=$ const. are the lines of curvature. Now the lines of length zero are given by

that is,

$$
d u_{1}^{2}+d v_{1}^{2}=0
$$

$$
u_{1}+i v_{1}=2 u, \quad u_{1}-i v_{1}=2 v,
$$

where $u$ and $v$ are the parameters of the lines of length zero. From (7) it follows that, if the surface is referred to lines of length zero, the equation of the lines of curvature is

$$
d u^{2}-d v^{2}=0
$$

and for a general choice of parameters this would be

$$
U d u^{2}-V d v^{2}=0,
$$

where $U$ is a function of $u$ alone and $V$ is a function of $v$ alone. Comparing this equation with equation (5) we get

$$
\frac{D}{D^{\prime \prime}}=\frac{U}{V}
$$

Hence

THEOREM IV. When an isothermic surface is referred to its lines of length zero, the lines of curvature are given by quadratures.

And conversely

Theorem V. When a surface is referred to its lines of length zero and the condition (8) is satisfied, the surface is isothermic.

In consequence of the preceding results the equation whose 
solution gives all surfaces of constant mean curvature can be reduced to a simple form. By a suitable choice of parameters we have $D=D^{\prime \prime}=1$, and there is no loss in assuming that the square of the mean curvature is unity. When these values are substituted in the Gauss equation,* it reduces to

$$
\frac{1}{F} \frac{\partial^{2} \log F}{\partial u \partial v}=\frac{1}{F^{2}}-1 \text {. }
$$

If we put $\log F=\theta$, this equation becomes

$$
\frac{\partial^{2} \theta}{\partial u \partial v}=e^{-\theta}-e^{\theta} .
$$

When a solution of this equation is known, all of the fundamental coefficients of the surface are given.

Princeton, N. J.,

September 20, 1902.

\section{SUPPLEMENTARY NOTE ON THE CALCULUS OF VARIATIONS.}

BY DR. E. R. HEDRICK.

IN a recent paper in the BuLletin, $\dagger$ the present writer stated the theorem: "The condition $\partial^{2} f / \partial y^{\prime 2}>0$ for all $x, y$ on the curve $C$, and for all $y^{\prime}$ considered, is a sufficient condition for a minimum" (of the integral $\int f\left(x, y, y^{\prime}\right) d x$ along the curve $C$ ), provided that certain preliminary requirements are satisfied. This theorem stands in apparent contradiction with an example given by Professor Bolza ; $\ddagger$ and while the contradiction is only apparent, it seems fitting to point out clearly the actual agreement of my results with those of Professor Bolza.

In the article mentioned I have required (page 11) that the integral $\int f\left(x, y, y^{\prime}\right) d x$, shall be considered only in a region $R$ (of the $x, y, y^{\prime}$ space), such that the integrand $f\left(x, y, y^{\prime}\right)$ is

* Bianchi, l. c., p. 67.

† "On the sufficient conditions in the calculus of variations," BuLletin, vol. 9 (2), no. 1 (Oct., 1902), p. 15.

$\ddagger$ "Some instructive examples in the calculus of variations," BULLETIN, vol. 9 (2), no. 1 (Oct., 1902), Example II, p. 9. 